\title{
Clinical Study on the Effect of Whole Course Nursing Intervention on Critically III Patients and the Occurrence of Complications
}

\author{
SHU YUAN LI, YAN YU, S. J. ZHEN, Z. J. XING AND XIN GAI* \\ Department of Intensive Care Unit, Shengli Oilfield Central Hospital, No. 31, Jinan Road, Dongying, Shandong 257034, China
}

\section{Li et al.: Effect of Whole Course Nursing Intervention on Critically Ill Patients}

\begin{abstract}
To explore the clinical effect of whole course nursing intervention on critically ill patients and control of complications is the main objective. The clinical data of $\mathbf{8 6}$ patients in intensive care unit of our hospital from January 2019 to June 2020 were selected and divided into two groups according to different nursing schemes, 43 cases in the control group and 43 cases in the study group. The control group was given routine nursing, while the study group was given comprehensive nursing, mainly including risk assessment, psychological nursing and life nursing. The psychological status, sleep quality, inflammatory factors, complications and nursing satisfaction of patients after nursing intervention were evaluated. There was no significant difference in self-rating anxiety scale, self-rating depression scale score and sleep quality score between the two groups before treatment $(p>0.05)$. After nursing intervention, self-rating anxiety scale, self-rating depression scale and sleep quality scores of the two groups were significantly lower than those of the control group, and the difference was statistically significant $(p<0.05)$. In addition, there was no significant difference in serum procalcitonin and interleukin- 6 levels between the two groups before treatment ( $p>0.05)$, after nursing, the serum procalcitonin and interleukin-6 levels of the study group were significantly lower than those of the control group $(p<0.05)$. Finally, the nursing satisfaction of the study group was significantly higher than that of the control group, and the infection and ventilator-associated pneumonia of the control group were higher than those of the study group $(\mathbf{p}<0.05)$. The implementation of scientific and effective whole course nursing intervention measures for severe patients can effectively alleviate their psychological state, improve the sleep quality of patients, reduce the level of inflammatory factors of patients, and then improve the nursing satisfaction, which has clinical application value.
\end{abstract}

Key words: Critical illness, whole course nursing, psychological nursing, complications

Critically ill patients are complex and rapid progress can cause serious threat to the lives of patients, is a common intensive care unit (ICU) patient. Due to the critical condition, patients and their families are in a high state of tension. It is very easy to have doctorpatient disputes, which has a negative impact on the rescue and treatment of patient ${ }^{[1,2]}$. ICU is an intensive care unit set up for critically ill patients with complete rescue equipment ${ }^{[3,4]}$. Relevant studies ${ }^{[5]}$ show that nearly $50 \%$ of severe patients may have negative psychological reactions such as negative, manic, anxiety, and most patients are prone to sleep disorders such as easy to wake up and insomnia, which will affect their recovery effect. Therefore, the implementation of effective nursing intervention for critically ill patients is of great significance ${ }^{[6]}$. In recent years, our hospital has implemented the whole process of nursing intervention in the emergency treatment of critically ill patients in the intensive care department, and achieved remarkable results. The results are as follows. A total of 86 patients in ICU of our hospital from January 2019 to June 2020 were selected and divided into two groups according to different nursing plans. The control group had 43 patients, including 25 males and 19 females, with an age range of 37-74 y (average 48.65 $44.32 \mathrm{y}$ ). According to the etiology, there were 15 cases of shock, 13 cases of severe pneumonia, 10 cases of heart failure and 5 cases of multiple-trauma. There were 43 patients in the study group, including 24 males and 20 females, with an average age of $48.18 \pm 3.87 \mathrm{y}$ old ranging from 38 to $73 \mathrm{y}$ old. According to the etiology, there were 16 patients with shock, 14 patients with severe pneumonia, 9 patients with heart failure and 4 patients with multiple-trauma. There was no significant 
difference in age, gender, etiology classification and other general information between the two groups $(\mathrm{p}>0.05)$. Control group: In this study, routine nursing methods were given to patients in the control group, mainly including electrocardiogram (ECG) examination, psychological counseling and medication on time, massage and regular inspection. Study group: To better explore the effect of comprehensive nursing intervention, this study on the observation group of patients with routine nursing at the same time, further give its comprehensive nursing intervention measures, including the following: Risk assessment: After admission, the relevant nursing staff should timely understand the patient's age, condition, ICU monitoring time and whether to use ventilator for treatment, and evaluate the possible risk factors of patients. For some elderly patients, we should strengthen the care and guidance to ensure that they can feel the importance, so that they can maintain a good treatment attitude ${ }^{[7]}$. For some high-risk patients, the corresponding safety emergency plan should be developed to ensure that patients can effectively prevent ICU syndrome and provide guarantee for the life safety of patients ${ }^{[8]}$. Psychological nursing: ICU patients with severe illness are relatively complex, when they are transferred to the ICU ward, they will be aware of the seriousness of their illness, so they are more prone to anxiety. Therefore, the relevant nursing staff should strengthen the psychological comfort of patients. For some conscious patients, nursing staff should communicate with them in time, understand the needs of patients in time, establish a good nurse patient relationship, and instruct patients not to worry too much, and actively co-operate with the treatment, so as to improve the treatment compliance of patients and effectively relieve the psychological pressure of patients. Life care: For patients, who cannot eat input nutrient solution to ensure that they can get sufficient nutrition. If patients can eat, then according to the actual situation of patients, carry out scientific diet guidance ${ }^{[9]}$. In addition, give patients a warm and comfortable ward environment, ensure the clean and tidy ward, help patients regulate their emotions, reduce their depression, anxiety and other negative emotions. Finally, play some soothing and relaxing music for patients to relieve their bad emotions. Compared the complications between the two groups, including catheter-related pneumonia, ventilator-associated pneumonia (VAP), deep venous catheter-related infection. Self-rating anxiety scale (SAS) and self-rating depression scale (SDS) were used to evaluate the psychological status of the patients, the higher the score, the higher the degree of depression and anxiety. According to Pittsburgh sleep quality index (PSQI) ${ }^{[10]}$, sleep quality was evaluated, including sleep time, sleep efficiency and sleep quality, the higher the score, the lower the sleep quality. Compared the inflammation and infection of the two groups, including the changes of serum inflammatory factor high sensitivity procalcitonin (PCT) and interleukin-6 (IL-6). The degree of satisfaction was divided into three grades: satisfaction $>90$, general satisfaction $60 \sim 90$ and dissatisfaction $<60 . \quad$ The total satisfaction $=($ satisfaction + general atisfaction/total cases) $\times 100 \%$. The data were analyzed by SPSS 22.0 statistical software, and the measurement data were expressed as $\mathrm{X} \pm \mathrm{s}$ by $\mathrm{t}$ test. The counting data were expressed as number of cases (n) and percentage (\%). $\mathrm{X}^{2}$ test was used for comparison among groups. $\mathrm{p}<0.05$ was considered statistically significant. As shown in Table 1, there was no significant difference in SAS and SDS scores between the two groups before treatment $(p>0.05)$. After nursing intervention, the SAS and SDS scores of the two groups were significantly decreased, and the study group was more significant than the control group $(p<0.05)$. Before treatment, there was no significant difference in sleep time, sleep efficiency and sleep quality between the two groups $(p>0.05)$. After treatment, the two groups were significantly improved, and the sleep time, sleep efficiency and sleep quality of the study group were significantly lower than those of the control group $(p<0.05)$, as shown in Table 2 . Before

\begin{tabular}{|c|c|c|c|c|}
\hline Group & $\mathbf{N}$ & Time & SAS score & SDS score \\
\hline \multirow[t]{2}{*}{$\begin{array}{l}\text { Control } \\
\text { group }\end{array}$} & 43 & Before treatment & $53.93 \pm 4.77$ & $57.61 \pm 6.33$ \\
\hline & & After treatment & $43.50 \pm 5.22^{*}$ & $45.59 \pm 6.03^{*}$ \\
\hline \multirow[t]{2}{*}{$\begin{array}{l}\text { Study } \\
\text { group }\end{array}$} & 43 & Before treatment & $52.71 \pm 4.89$ & $58.82 \pm 6.93$ \\
\hline & & After treatment & $30.18 \pm 3.27^{*} \#$ & $32.26 \pm 5.16^{*} \#$ \\
\hline
\end{tabular}

Note: Compared with before treatment, ${ }^{*} p<0.05$, compared with the control group, $\# \mathrm{p}<0.05$

TABLE 2: COMPARISON OF SLEEP QUALITY BETWEEN THE TWO GROUPS

\begin{tabular}{lccccc}
\hline Group & N & Sleep latency & Sleep time & Sleep efficiency & Sleep quality \\
\hline Study group & 43 & $1.26 \pm 0.43 \#$ & $0.78 \pm 0.35 \#$ & $0.57 \pm 0.21 \#$ & $1.02 \pm 0.41 \#$ \\
Control group & 43 & $1.92 \pm 0.66$ & $1.37 \pm 0.54$ & $0.93 \pm 0.35$ & $1.64 \pm 0.55$ \\
\hline
\end{tabular}

Note: Compared with the control group, $\# p<0.05$ 
treatment, there was no significant difference in serum PCT and IL-6 levels between the two groups ( $p>0.05)$; after nursing, serum PCT and IL-6 levels in the study group were significantly lower than those in the control group $(p<0.05)$ (Table 3$)$. The results showed that the nursing satisfaction of the study group was significantly higher than that of the control group $(\mathrm{p}<0.05)$ (Table 4). During the treatment, the incidence of catheter-related infection and VAP in the control group was higher than that in the study group $(\mathrm{p}<0.05)$ as shown in Table 5 . ICU as a special place to treat critically ill patients, serious illness, low body resistance and immunity, and poor self-care ability, which is prone to accidents in nursing work and affects the quality of nursing ${ }^{[11-13]}$. In addition, in severe patients, the condition is critical and the disease is more complex, so multiple items need to be monitored. Therefore, the implementation of whole course nursing intervention for critically ill patients, the optimization of human resource allocation in nursing work, and the implementation of whole course nursing for patients can effectively improve the quality of nursing, shorten the length of hospital stay, and reduce economic pressure. ICU patients are seriously ill, facing the threat of life, their psychology is in a high state of stress and the environment of intensive care is relatively closed. In addition, the medical staff only follows the doctor's advice objectively and do not actively communicates with the patients, so it is easy to ignore their emotional needs. In addition, patients are prone to bad emotions when they are in a state of depression for a long time ${ }^{[13-15]}$. Therefore, the clinical need is to take effective and reasonable nursing intervention measures to alleviate the psychological state and sleep disorders of patients, so as to achieve the purpose of improving the curative effect. In this study, we compared and analyzed the nursing effect of routine nursing mode and whole course nursing intervention on patient's psychological state and sleep quality. The results showed that two groups of patients after nursing SAS and SDS scores were significantly lower than before nursing and the study group was significantly lower than the control group. Meanwhile, the sleep time and sleep time scores of the study group were lower than those of the control group, and the nursing satisfaction $(90.70 \%)$ was higher than that of the control group (76.74\%), suggesting that the whole process nursing intervention measures applied to ICU patients cannot only improve their mental state and sleep disorders, but also effectively improve the quality of life, nursing satisfaction. The whole process nursing intervention program of the research group comprehensively considered the psychological state changes of patients in the process of treatment, adopted scientific psychological suggestion, and actively guided them to establish the confidence to overcome the disease. According to the different types of patients with different causes, the guidance of diet and medication was given, and some health knowledge education and publicity activities were carried out from time to time. The patients were instructed to persist in functional exercise, maintain a positive and optimistic attitude, co-operate with treatment and nursing, so as to speed up the rehabilitation process. In addition, we have strengthened mutual communication with patients, established a benign interactive channel, given more care and care to patients, so as to reduce their loneliness, further improve patients nursing and treatment

TABLE 3: COMPARISON OF INFLAMMATORY FACTORS BETWEEN THE TWO GROUPS

\begin{tabular}{lccccc}
\hline \multirow{2}{*}{ Group } & $\mathrm{N}$ & \multicolumn{2}{c}{$\mathrm{PCT}(\mathrm{ng} / \mathrm{ml})$} & \multicolumn{2}{c}{$\mathrm{IL}-6(\mathrm{pg} / \mathrm{ml})$} \\
\cline { 3 - 6 } & & Before treatment & After treatment & Before treatment & After treatment \\
Study group & 43 & $3.45 \pm 0.83$ & $1.52 \pm 0.54^{*} \#$ & $65.27 \pm 10.26$ & $34.56 \pm 5.38^{*} \#$ \\
Control group & 43 & $3.41 \pm 0.82$ & $1.89 \pm 0.63^{*}$ & $64.87 \pm 11.01$ & $39.53 \pm 6.92^{*}$ \\
\hline
\end{tabular}

Note: Compared with before treatment, ${ }^{*} p<0.05$, compared with the control group, $\# p<0.05$

TABLE 4: COMPARISON OF INFLAMMATORY FACTORS BETWEEN THE TWO GROUPS

\begin{tabular}{lccccc}
\hline Group & N & Satisfied & Generally satisfied & Dissatisfied & Degree of satisfaction \\
\hline Study group & 43 & 16 & 23 & 4 & $39(90.70 \%) \#$ \\
Control group & 43 & 11 & 21 & 10 & $10(76.74 \%)$ \\
\hline
\end{tabular}

Note: Compared with the control group, \#p<0.05

TABLE 5: COMPARISON OF COMPLICATIONS BETWEEN THE TWO GROUPS

\begin{tabular}{lcccccc}
\hline Group & N & Catheter indwelling pneumonia & Deep venous catheter infection & VAP & $\begin{array}{c}\text { Incidence of } \\
\text { complications }\end{array}$ \\
\hline Study group & 43 & 1 & 0 & 2 & $3(6.98 \%) \#$ \\
Control group & 43 & 2 & 1 & 7 & $10(23.26 \%)$ \\
\hline
\end{tabular}

Note: Compared with the control group, $\# p<0.05$ 
cooperation, and then significantly improve sleep quality ${ }^{[16]}$. Severe systemic infection is common in ICU patients during treatment. PCT and IL-6 are important inflammatory factors, which mediate the pathological damage of some autoimmune diseases ${ }^{[17]}$. The results showed that before the nursing intervention, there was no significant difference in the levels of serum PCT and IL-6 inflammatory factors between the two groups ( $p>0.05$ ); after the nursing, the levels of serum PCT and IL-6 in the study group were significantly lower than those in the control group, indicating that the whole process nursing intervention can reduce the expression levels of serum PCT and IL-6 inflammatory factors, and promote the recovery of patients as soon as possible. At the same time, in the study, it was found that the infection and VAP in the control group were higher than those in the study group $(\mathrm{p}<0.05)$, which also showed that the whole process nursing intervention was beneficial to reduce the complications of patients. In conclusion, the implementation of scientific and effective whole process nursing intervention measures for ICU patients can effectively alleviate their psychological state, improve the sleep quality of patients, reduce the level of inflammatory factors of patients, and then improve nursing satisfaction, which has clinical application value.

\section{Acknowledgements:}

This study was supported by the Shengli Oilfield Central Hospital.

\section{Conflict of Interests:}

The authors declared no conflict of interest.

\section{REFERENCES}

1. Lam S, Scannell R, Roessler D, Smith MA. Peripherally inserted central catheters in an acute-care hospital. Arch Intern Med 1994;154:1833-7.

2. Crowley JJ. Vascular access. Tech Vasc Interv Radiol 2003;6:176-81.

3. Tzeng HM. Roles of nurse aides and family members in acute patient care in Taiwan. J Nurs Care Qual 2004;19:169-75.

4. Hogan MJ. Neonatal vascular catheters and their complications. Radiol Clin North Am 1999;37:1109-25.

5. Jasinsky LM, Wurster J. Occlusion reduction and heparin elimination trial using an antireflux device on peripheral and central venous catheters. J Infus Nurs 2009;32:33-9.

6. Ngo A, Murphy S. A theory-based intervention to improve nurses' knowledge, self-efficacy, and skills to reduce PICC occlusion. J Infus Nurs 2005;28:173-81.

7. Safdar N, Maki DG. Risk of catheter-related bloodstream infection with peripherally inserted central venous catheters used in hospitalized patients. Chest 2005;128:489-95.

8. Cheong K, Perry D, Karapetis C, Koczwara B. High rate of complications associated with peripherally inserted central venous catheters in patients with solid tumours. Intern Med J 2004;34:234-8.

9. Yamamoto AJ, Solomon JA, Soulen MC, Tang J, Parkinson $\mathrm{K}$, Lin $\mathrm{R}$, et al. Sutureless securement device reduces complications of peripherally inserted central venous catheters. J Vasc Interv Radiol 2002;13:77-81.

10. Walshe LJ, Malak SF, Eagan J, Sepkowitz KA. Complication rates among cancer patients with peripherally inserted central catheters. J Clin Oncol 2002;20:3276-81.

11. Marnejon T, Angelo D, Abdou AA, Gemmel D. Risk factors for upper extremity venous thrombosis associated with peripherally inserted central venous catheters. J Vasc Access 2012;13:231-8.

12. Chow LM, Friedman JN, MacArthur C, Restrepo R, Temple M, Chait PG, et al. Peripherally inserted central catheter (PICC) fracture and embolozation in the pediatric population. J Pediatr 2003;142:141-4.

13. Loewenthal MR, Dobson PM, Starkey RE, Dagg SA, Petersen A, Boyle MJ. The peripherally inserted central catheter (PICC): a prospective study of its natural history after cubital fossa insertion. Anaesth Intensive Care 2002;30:21-4.

14. Racadio JM, Doellman DA, Johnson ND, Bean JA, Jacobs BR. Pediatric peripherally inserted central catheters: complication rates related to catheter tip location. Pediatrics 2001;107:e28.

15. Amerasekera SS, Jones CM, Patel R, Cleasby MJ. Imaging of the complications of peripherally inserted central venous catheters. Clin Radiol 2009;64:832-40.

16. Dubois J, Garel L, Tapiero B, Dube J, Laframboise S, David M. Peripherally inserted central catheters in infants and children. Radiology 1997;204:622-6.

17. Vidal V, Muller C, Jacquier A, Giorgi R, Le Corroller T, Gaubert J, et al. Prospective evaluation of PICC line related complications. J Radiol 2008;89:495-8.

This is an open access article distributed under the terms of the Creative Commons Attribution-NonCommercial-ShareAlike 3.0 License, which allows others to remix, tweak, and build upon the work non-commercially, as long as the author is credited and the new creations are licensed under the identical terms

This article was originally published in a special issue,
"Evolutionary Strategies in Biomedical Research and
Pharmaceutical Sciences" Indian J Pharm Sci 2020:83(3)
Spl issue;73-76

